3bändige Werk für den praktischen Arzt und Studirenden als sehr brauchbar zu empfehlen.

\title{
Varia.
}

Geh. Rath Prof. Dr. G. Lewin war in den letzten Tagen anlässlich seines 50jäbrigen Doctorjubiläums Gegenstand auszeichnender Ehrungen, welche Zengniss ablegten für die ausserordentliche Hochachtung und Wertbschätzung, deren sich der Gefeierte in den weitesten wissenschaftlichen und ärztlichen Kreisen erfreut. Es ist selbstverständlich, dass nach dieser Richtung die wissenschaftlichen und ärztlichen Körperschaften Berlins, der Stätte, an welcher der gefeierte Jubilar durch ein halbes Jahrbundert seine segensreiche Thätigkeit entfaltete, vorangingen.

Am 7. November wurde der Jubilar durch zahlreiche Abordnungen der Berliner Gesellschaften und Vertreter der Institute, an denen Prof. L e w in unermüdlich thätig ist, beglückwünseht.

In solenner Weise veranstaltete die Berliner dermatologische Vereinigung am 12. d. M. zu Ehren Lewin's eine Festsitzung in den Räumen des Langenbeck-Hauses. An die Begrüssung des Jubilars durch den zweiten Vorsitzenden, Herrn Prof. Lassar schloss sich die Ueberreichung einer Festschrift, für welche 21 Freunde, Schüler und Fachgenossen $I_{s}$ ewin's Beiträge geliefert baben, die zusammen einen im Verlage von Karger erschienenen stattlichen Band bilden. Hierauf folgten wissenschaftliche Vorträge der Herren Virchow und Leyden und zahlreiche Demonstrationen von Präparaten und Objecten aus dem Gebiete der Dermatologie.

Berichtigung. Ohne irgendwie Unna's Verdienste um unsere Kenntnisse über die Ulcus molle-Mikroben reduciren zu wollen, kann ich es doch nicht unterlassen, eine Ungenauigkeit in seinem Aufsatze: „Die verschiedenen Phasen des Streptobacillus Ulceris mollis", Monatsh. f. prakt. Dermatol. Bd. 21, Nr, 2 dieses Jahres zu erwähnen.

Unna will nämlich in obengenannter Abhandlung meine Hauptarbeit betreffs Ulcus molle so datiren, als wäre sie $\mathrm{nach}$ seinem Artikel "Der Streptobacillus des weichen Schankers", Monatshefte für prakt. Dermatol., Julibeft 1892 geschrieben. Er erwähnt nämlich nur die deutsche Uebersetzung meiner Arbeit in diesem Archiv 1892, während meine erste Mittheilung bereits im Jahre 1891 in Nord. med. Archiv (Resumé in französischer Sprache) erschienen ist. Ausserdem dass ich 
Hrn. Unna, wie ich mich bestimmt erinnere, einen Separatabdruck hievon gesandt habe, ist auch in meinen beiden späteren Aufsätzen in Annales de Dermatologie et syphil. 1893 darauf hingewiesen. Aus einem dieser Aufsätze hat Unna wie man sieht, mehrere Zeilen auf französisch eitirt, so dass er auch auf die darin erwähnte Arbeit von 1891 hätte aufmerksam werden müssen.

Nach Unna sieht es demnach so aus, als ob zwischen Duerey und ihm kein Verfasser existiren würde, der über diesen Gegenstand etwas publicirt hätte, während es in Wirklichkeit sich so verhält, dass D u crey's Arbeit von 1889, die meinige von 1891 und Unna's über ein halbes Jahr später von 1892 datirt.

Christiania, 16. Sept. 1895.

\section{Rudolf Krefting.}

